\title{
Efficacy and Safety of Postoperative Dexmedetomidine Administration in Infants and Children Undergoing Cardiac Surgery: A Retrospective Cohort Study
}

\author{
Robert Horvath $^{1}$ Emma F. Halbrooks ${ }^{2}$ David M. Overman ${ }^{1,2}$ Stefan J. Friedrichsdorf ${ }^{3,4}$ \\ ${ }^{1}$ Children's Heart Clinic, Children's Hospitals and Clinics of Minnesota, \\ Minneapolis, Minnesota, United States \\ ${ }^{2}$ Critical Care and Cardiovascular Research Center, Children's \\ Hospitals and Clinics of Minnesota, Minneapolis, Minnesota, United \\ States \\ ${ }^{3}$ Department of Pain Medicine, Palliative Care and Integrative \\ Medicine, Children's Hospitals and Clinics of Minnesota, Minneapolis, \\ Minnesota, United States \\ ${ }^{4}$ Department of Pediatrics, University of Minnesota Medical School, \\ Minneapolis, Minnesota, United States \\ J Pediatr Intensive Care 2015;4:138-145. \\ Address for correspondence Robert Horvath, MD, Children's Heart \\ Clinic, Children's Hospitals and Clinics of Minnesota, 2530 Chicago \\ Avenue S. CSC-500, Minneapolis, MN 55404, United States \\ (e-mail: RHorvath@chc-pa.org).
}

\begin{abstract}
\section{Keywords}

- dexmedetomidine

- pediatric cardiac surgery

- pain

- sedation

- trisomy 21

- withdrawal

In this retrospective cohort study, we describe efficacy and safety of dexmedetomidine administration in the cardiac intensive care unit, in children undergoing cardiac surgery. This study consisted of pediatric patients 3 days to 17.5 years of age, undergoing cardiac surgery from April 2010 to April 2011. In addition to a scheduled opioid, usually fentanyl, 107 patients received dexmedetomidine postoperatively, including 19 (18\%) patients diagnosed with trisomy 21 and $9(8 \%)$ neonates/newborns. Median dexmedetomidine starting dose was $0.45 \mu \mathrm{g} / \mathrm{kg} / \mathrm{h}$ and overall median dose higher than previously published, ranging from $0.8 \mu \mathrm{g} / \mathrm{kg} / \mathrm{h}$ to $2.17 \mu \mathrm{g} / \mathrm{kg} / \mathrm{h}$. We compared infants (younger than 1 year) with older children (1-17 years of age). Median duration of administration was 1.6 days (0-23.9 days) and duration to extubation in infants was 46.5 hours, and in the older children it was 22.6 hours. Seven patients (6.5\%) were discontinued dexmedetomidine due to adverse events, most commonly bradycardia. Neonates experienced more pronounced heart rate reduction (no bradycardia) and received lower maintenance doses than older infants ( $0.56 \mathrm{vs} .0 .77 \mu \mathrm{g} / \mathrm{kg} / \mathrm{h}$ ). The face, leg, activity, cry, consolability pain scale and the state behavioral scale scores indicated adequate sedation and good analgesia. The most common adverse reaction postdiscontinuation was agitation, more frequent in the infant group (20 vs. $11.2 \%$ ). Thirty patients $(28.3 \%$ ) received prolonged infusion ( $>72$ hours). Patients with and without trisomy 21 did not differ with dose, safety, efficacy, or adverse events. Dexmedetomidine, as part of multimodal management, appears to be a safe, efficacious agent providing analgesia and sedation throughout all pediatric age groups following cardiac surgery. Overall, dexmedetomidine is well tolerated and safe with higher doses than previously noted, and is well tolerated by neonates, infants, and patients with trisomy 21. Withdrawal effects were noted in patients following prolonged infusion.
\end{abstract}

received

June 27, 2014

accepted after revision

March 5, 2015

published online

August 12, 2015
Copyright $\odot 2015$ by Georg Thieme

Verlag KG, Stuttgart · New York
DOI http://dx.doi.org/ 10.1055/s-0035-1559820. ISSN 2146-4618. 


\section{Introduction}

Management of postoperative pain and sedation remains challenging in the pediatric cardiovascular intensive care units (CVICU), especially in neonates, infants, children with chromosomal abnormalities, and children who require longterm intubation. In recent years, the $\alpha$-2-adrenergic agonist dexmedetomidine gained prominence as part of a multimodal (opioid- and benzodiazepine-sparing) analgesic and sedative approach. Currently, the Food and Drug Administration (FDA) in the United States approves a dexmedetomidine loading dose of $1 \mu \mathrm{g} / \mathrm{kg}$ and a maintenance infusion of 0.2 to $0.7 \mu \mathrm{g} / \mathrm{kg} / \mathrm{h}$ for up to 24 hours in adults. ${ }^{1}$ Dexmedetomidine in our clinical experience and according to published data has been effective as an analgesic as well as sedative. The off-label use of this medication has expanded significantly over the past 10 years in pediatric intensive care units. ${ }^{2-4}$

The cardiac surgeons at Children's Hospitals and Clinics of Minnesota in Minneapolis perform more than 400 heart surgeries in infants and older children annually, and a significant number of patients receive dexmedetomidine infusion postoperatively in the CVICU. Due to the nature of the patient population in the CVICU, use of dexmedetomidine often falls outside the standard application, including higher dosage than FDA approval, younger age (including neonates), and prolonged use of an infusion greater than 72 hours. The aim of this retrospective study was to evaluate efficacy, safety, and tolerability of dexmedetomidine administration in pediatric patients less than 18 years of age.

\section{Materials and Methods}

This Institutional Review Board-approved study consisted of a single-site retrospective review of pediatric patients admitted to the CVICU at Children's Hospitals and Clinics of Minnesota between April 2010 and April 2011, who received dexmedetomidine postoperatively. Due to the retrospective nature of the study, the Institutional Review Board waived the need for informed consent. We collected demographic information including age, gender, weight, cardiac diagnosis, surgical procedure, risk adjustment for congenital heart surgery (RACHS) score, date and time of postoperative CVICU arrival, and comorbid genetic diagnoses, specifically trisomy $21 .^{5}$

Length of dexmedetomidine administration, as well as bolus doses, and daily mean and maximum infusion doses were recorded. Intubation status at start of drug administration, as well as date and time of extubation, and any adverse effect (clinically significant high or low blood pressure, bradycardia, and low respiratory rate \pm 2 standard deviation from normal for the age group) resulting in discontinuation of dexmedetomidine were recorded.

We documented inotropic score for all patients, which reflect the patient's inotropic needs postoperatively. ${ }^{6}$

To assess safety and possible withdrawal effects, heart rate, respiratory rate, and systolic and diastolic blood pressure were recorded prior to administration, and at hour 12 and 24 postinitiation. Daily minimum, maximum, and mean values up to day 5 were also collected. Any use of enteral/transdermal clonidine to help treat withdrawal following discontinuation of dexmedetomidine was recorded. In the United States, clonidine is not approved for use in intravenous formulation.

As a part of CVICU protocol, if a scheduled opioid (usually fentanyl) plus simple analgesia (acetaminophen and/or nonsteroidal anti-inflammatory drug) did not provide appropriate analgesia and sedation, we added dexmedetomidine. Efficacy of dexmedetomidine as part of a multimodal analgesia and sedation protocol was reviewed by collecting the face, leg, activity, cry, consolability (FLACC) pain scale (0 to 10) and the state behavioral scale ([SBS]; -2 to +2 ) for sedation starting prior to dexmedetomidine administration and continuing until discontinuation; we also noted if any symptoms of delirium or agitation were documented by the nurse or physicians. ${ }^{7,8}$ Concomitant medications used were also noted, specifically opioids (fentanyl, morphine, oxycodone, and methadone), benzodiazepines (lorazepam and midazolam), and general anesthetics (propofol and ketamine), noting whether those medications were used prior to, concurrent with, or postadministration of dexmedetomidine.

Statistical analysis included frequency (percentage) for categorical variables, and comparisons utilizing chi-squared test or Fisher exact test, as needed. Mean (standard deviation) described continuous variables, and median (range) for significantly skewed variables. $t$-test or Mann-Whitney compared age subgroups, and the Friedman test provided comparisons for vital sign data to baseline within age subgroups. The Wilcoxon signed ranked test compared daily data to baseline for sample sizes over 15 . A 95\% confidence interval was calculated using two standard errors and $p$-values $<0.05$ were considered significant. (Statistical analysis was conducted with Statistical Package for the Social Sciences 15.0, Chicago, Illinois, United States.)

\section{Results}

From April 2010 to April 2011, a total of 403 children younger than 18 years underwent cardiac surgery and received postoperative care in our CVICU. Of those, 107 patients (26.5\%), ages ranging from 0.1 month to 17.5 years, received a dexmedetomidine infusion and were included in our study. Initiation of dexmedetomidine was based on the judgment of the bedside intensivist. Twenty-one (19.6\%) patients received a bolus dose. We analyzed and compared infants (less than 1 year) to older children (older than 1 year) separately. - Table 1 summarizes the patients' overall characteristics. Of the 107 patients enrolled, 54.2\% were male and $19(17.8 \%)$ were diagnosed with trisomy 21.

\section{Concurrent Analgesics/Sedatives}

During dexmedetomidine infusion, other medications were administered to provide postoperative analgesia and sedation and six were analyzed in this study group; three opioids (fentanyl, morphine, and oxycodone), the benzodiazepines (lorazepam and midazolam), and the sedative/hypnotic (propofol). Of the 107 patients included, only 2 patients ( $1.9 \%$ ) received none of the other five noted 
Table 1 Overall patient characteristics

\begin{tabular}{|l|l|}
\hline Characteristics & Median (range) \\
\hline Number & 107 \\
\hline Age $(\mathrm{mo})$ & $6.2(0.1-209.4)$ \\
\hline Weight $(\mathrm{kg})$ & $6.5(3-77.3)$ \\
\hline Risk adjustment for congenital heart surgery score $(1-6)$ & $3(1-6)$ \\
\hline Dexmedetomidine starting dose $(\mu \mathrm{g} / \mathrm{kg} / \mathrm{h})$ & $0.45(0.1-2.17)$ \\
\hline Dexmedetomidine bolus dose $(\mu \mathrm{g} / \mathrm{kg})$ & $0.99(0.12-2.00)$ \\
\hline Length of dexmedetomidine administration $(\mathrm{d})$ & $1.6(0.0-230.9)$ \\
\hline $\begin{array}{l}\text { Time interval of dexmedetomidine administration } \\
\text { following postoperative CVICU admission (h) }\end{array}$ & $16.8(0-181.8)$ \\
\hline Extubation after CVICU (h) & $22.6(0.5-619.8)$ \\
\hline & $\mathrm{Number} \mathrm{( \% )}$ \\
\hline Gender: male & $58(54.2)$ \\
\hline Neonates $0-1$ mo & $9(8.4)$ \\
\hline Age groups & \\
\hline$\leq 1$ y old & $70(65.4)$ \\
\hline$>1$ y old & $37(34.6)$ \\
\hline Intubated when dexmedetomidine started & $69(64.5)$ \\
\hline Dexmedetomidine bolus given & $21(19.6)$ \\
\hline Onset of delirium prior to dexmedetomidine administration & $9(8.4)$ \\
\hline Onset of agitation prior to dexmedetomidine administration & $14(13.1)$ \\
\hline Trisomy 21 & $19(17.8)$ \\
\hline Other syndromes ${ }^{\mathrm{a}}$ & $16(15.0)$ \\
\hline
\end{tabular}

Abbreviation: CVICU, cardiovascular intensive care unit.

${ }^{a}$ Other syndromes included DiGeorge $(n=5)$, Williams syndrome, Shone-like variant, partial trisomy 18, partial monosomy chromosome 10 and partial trisomy 12, Kabuki syndrome, autism, 22 microdeletion syndrome, and CHARGE syndrome.

concomitant medications. Overall, 39.3\% (42) received one of the listed medications along with dexmedetomidine, $31.8 \%$ (34) received two, $18.7 \%$ (20) three, and $6.6 \%(7)$ of patients received four or five of the listed medications concurrently with dexmedetomidine.

Most commonly, patients received fentanyl along with dexmedetomidine (86\%, or 92 patients), and of those 92 patients, $56.5 \%$ (52) received fentanyl within 24 hours prior to dexmedetomidine, during administration, and within 24 hours postadministration. Of the $39.3 \%$ of patients (42) who received only one other listed concurrent medication during dexmedetomidine administration, 34 patients received fentanyl. Of the $31.8 \%$ of patients who received two of the five listed concurrent medications, two combinations were the most common, fentanyl plus propofol and fentanyl plus oxycodone. No medications other than clonidine were used to wean off dexmedetomidine. Midazolam and lorazepam were used only in two patients, respectively (3.8\%).

\section{Efficacy}

To evaluate the effect of dexmedetomidine on respiratory rate, only nonintubated patients at initiation of dexmedetomidine were analyzed $(n=39)$. Infants showed the least change in mean respiratory rates from baseline, with mean respiratory rate remaining 31 to 34 breaths $/$ min $(p=0.655)$. Older patients showed the largest decrease from baseline 27 to 20 breaths/ $\min$ at hour $12(p=0.831)$. The mean respiratory rate on all extubated patients was 24 breaths/min (12-86) at baseline and remained stable throughout day $5(p=0.411)$. These changes were clinically and statistically not significant.

To assess the sedative and analgesic effect of dexmedetomidine as part of multimodal management in the CVICU, the documented scores of the FLACC scale and the SBS score were analyzed. One hundred and four patients (99\%) had documented FLACC scores and 42 patients (39\%) SBS scores. The mean FLACC scores were well below $2 / 10$ before and during the study period, with a statistically significant increase in predexmedetomidine administration compared with day 1 postinitiation from $0.88 / 10$ to $1.39 / 10$ ( - Table 2 ), indicating adequate analgesia. The SBS sedation scores showed no significant changes either prior to dexmedetomidine administration or after initiation $(p=0.22)$, indicating adequate level of sedation in our patients.

\section{Safety}

To assess overall safety, changes in vital signs throughout the administration of dexmedetomidine were analyzed and compared with age-appropriate standards. Blood pressure remained consistent across age groups and showed no 
Table 2 Pain and sedation scores by day

\begin{tabular}{|c|c|c|c|c|c|c|}
\hline Days & Day 0 (baseline) & Day 1 & Day 2 & Day 3 & Day 4 & Day 5 \\
\hline \multicolumn{7}{|c|}{ Pain scores: the face, leg, activity, cry, consolability $(0-10)$} \\
\hline Number & 104 & 92 & 96 & 54 & 35 & 27 \\
\hline Mean (standard deviation) & $0.88(1.06)$ & $1.39^{\mathrm{a}}(1.50)$ & $1.38(1.07)$ & $1.03(0.85)$ & $1.26(0.86)$ & $1.34(1.05)$ \\
\hline \multicolumn{7}{|c|}{ Sedation scores: the state behavioral scale $(-2$ to +2$)$} \\
\hline Number & 42 & 32 & 41 & 29 & 19 & 18 \\
\hline Mean (standard deviation) & $-0.88(0.84)$ & $-0.38(1.00)$ & $-0.44(0.60)$ & $-0.41(0.48)$ & $-0.29(0.43)$ & $-0.56(0.42)$ \\
\hline
\end{tabular}

${ }^{a}$ Statistically significant change $(p=0.004)$.

statistically significant changes. Of statistical significance, the average heart rate among infants decreased from 145 beats per minute (bpm) at baseline to $125.5 \mathrm{bpm}$ $(p=0.023)$, while older children had a decrease from

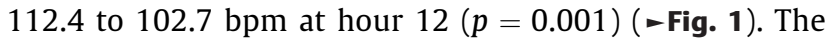
day-to-day heart rate remained unchanged through day 5 but decreased significantly from baseline $(p=0.023)$. Systolic blood pressure remained stable throughout the first 5 days of administration.

Time to extubation among the age groups (infants vs. older children) was statistically significant. Infants were intubated longer, with a median time of 46.5 hours (3.7$619.8)$; patients older than 1 year were extubated at a median time of 8.5 hours (0.5-134.9) after CVICU arrival $(p<0.001)$. Median duration of intubation for neonates (less than 1 month of age) was 72.3 hours. We have not detected respiratory depression.

\section{Inotrope Score}

We documented the inotrope score for all patients, to assure that level of sedation did not affect the hemodynamic stability of our patients. Inotrope scores in all patients decreased significantly at 24 hour from baseline $(p<0.001)$ and continued to decrease throughout day 5 ( - Fig. 2 ). The patients required progressively decreasing inotropic support throughout days 1 to 5 , while having stable hemodynamic parameters, reflected by normal systolic blood pressure and heart rate (-Fig. $\mathbf{1}$ ).

\section{Adverse Effects/Withdrawal}

Seven patients (6.5\%) experienced adverse reactions following dexmedetomidine initiation, identified as clinically significant high or low blood pressure, bradycardia, and low respiratory rate ( \pm 2 standard deviation from normal for the age group). Adverse reactions (hypotension and bradycardia)

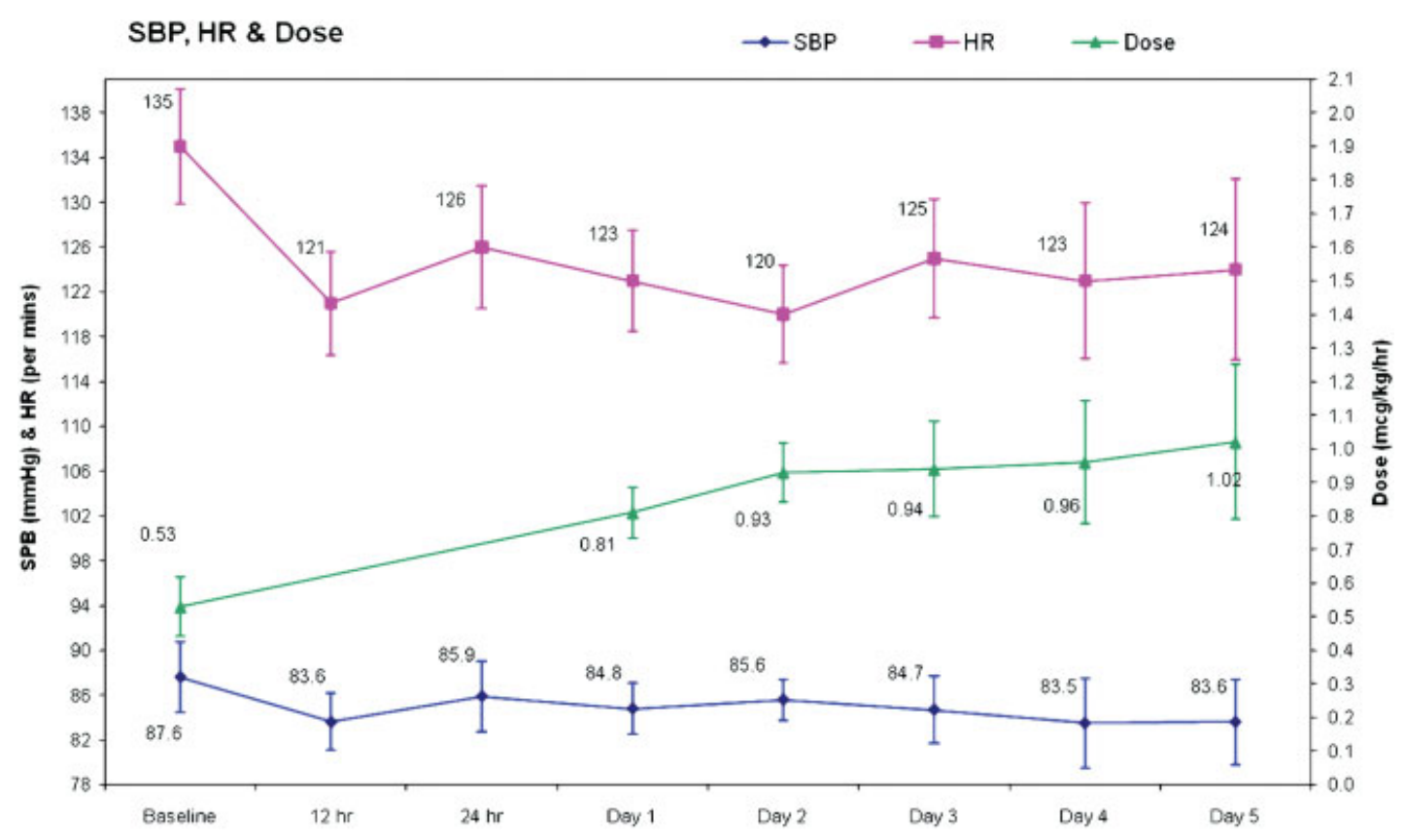

Fig. 1 Mean dose of dexmedetomidine, systolic blood pressure, and heart rate in all patients. Statistically significant decrease in heart rate between baseline and hour $12(p<0.001)$. No significant difference in systolic blood pressure from baseline. The bars in the graphs represent the $95 \%$ confident intervals for each point estimate. 


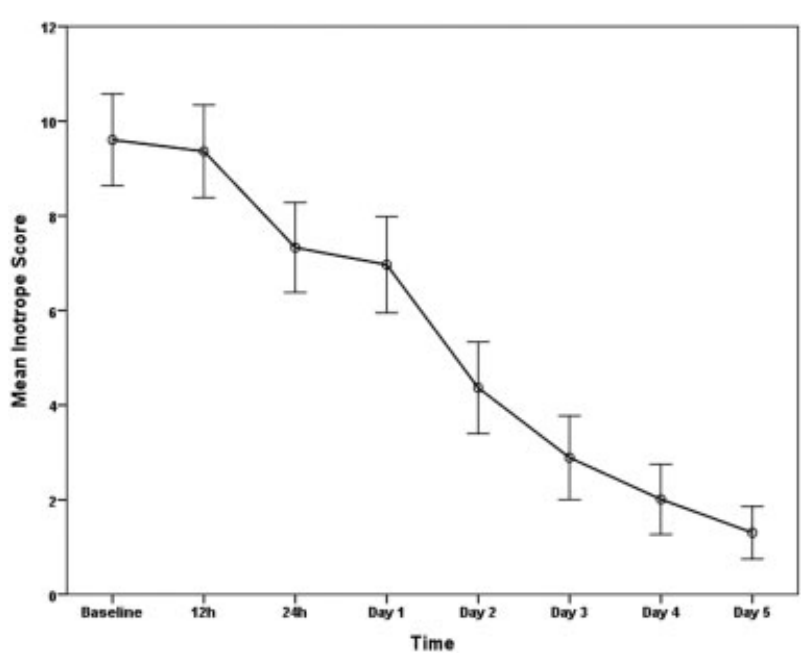

Fig. 2 Mean inotrope score for all patients. There is a statistically significant decrease from the baseline at 24 hour $(p<0.001)$. The bars in the graph represent the $95 \%$ confident intervals for each point estimate.

correlated with a higher median bolus of $1.06 \mu \mathrm{g} / \mathrm{kg}$ versus $0.21 \mu \mathrm{g} / \mathrm{kg}(p=0.04)$.

Twenty-three children (21.7\%) displayed clinical symptoms of withdrawal upon weaning and discontinuation of the study drug, defined as significant change in blood pressure (hypertension) and heart rate (rebound tachycardia), with increased agitation. Agitation after dexmedetomidine discontinuation was higher, but did not reach statistical significance $(p=0.27)$ in the infant group (-Table 3 ). Prolonged infusion, defined as greater than 72 hours of continuous infusion, resulted in higher risk for withdrawal symptoms (-Table 4).

\section{Infants}

The majority of our patients were infants (65\%). Comparing the infants (0-12-month-old group) to older children (older than 1 year), dexmedetomidine in infants was initiated later in the treatment trajectory (mean 20.8 vs. 8.3 hours postoperatively) and administered for a longer period of time (median 43.5 vs. 28 hours), and the infants were extubated later (46.5 vs. 8.4 hours postoperatively) (- Table 5 ).

On subgroup analysis of the infants, differences between the nine newborns (0-1 month) in comparison to older infants (1-12 months) did not reach statistical significance for the analyzed variables. Our statistical power was marginal on this patient group. Newborns had a median RACHS score of 3 (range: 1-6) and received a dexmedetomidine dose of 0.56 $\mu \mathrm{g} / \mathrm{kg} / \mathrm{h}$ (range: $0.35-1.05$ ), with infusion length of 1.8 days (0.9-15) and time to extubation of 72.3 hours (49.5-308.7).

Six neonates were extubated while on dexmedetomidine and three were extubated at the time of dexmedetomidine discontinuation. The baseline heart rate compared with heart rate 12 hours postinitiation changed from a mean of $157 \mathrm{bpm}$ to 127 bpm and remained stable for the duration of the administration. No adverse reactions led to discontinuation of dexmedetomidine among the neonates, and only one neonate exhibited agitation after discontinuation of the study drug.

\section{Children with Trisomy 21}

Nineteen (18\%) of the patients included in this study had a diagnosis of trisomy 21 . There were no significant differences between the patients with trisomy 21 and those without this chromosomal aberration with regard to age, weight, RACHS score, starting dose, duration on dexmedetomidine, or duration to extubation following admission to CVICU. No statistically significant differences existed between groups with regard to adverse responses or withdrawal syndrome. We excluded patients with "other" identifiable genetic syndromes from this analysis.

\section{Discussion}

This retrospective study describes a single institution's experience of using dexmedetomidine postoperatively as part of a

Table 3 Adverse reactions after initiation of dexmedetomidine and after discontinuation of dexmedetomidine

\begin{tabular}{|c|c|c|}
\hline Age groups & $\leq 1$ year old & $>1$ year old \\
\hline Number (\%) & $70(65 \%)$ & $37(35 \%)$ \\
\hline Adverse reactions & \multicolumn{2}{|l|}{ Number (\%) } \\
\hline Any adverse reactions & $4(5.7)$ & $3(16.4)$ \\
\hline Discontinued for high blood pressure & $1(1.5)$ & $0(0)$ \\
\hline Discontinued for low blood pressure & $0(0)$ & $1(5.6)$ \\
\hline Discontinued for low heart rate & $2(2.9)$ & $1(5.6)$ \\
\hline Discontinued for low respiratory rate & $0(0)$ & $1(5.3)$ \\
\hline Any adverse reaction after discontinuing dexmedetomidine & $17(24.3)$ & $6(16.3)$ \\
\hline Withdrawal syndrome & $4(5.8)$ & $2(5.6)$ \\
\hline Hypertension & $1(1.4)$ & $2(5.6)$ \\
\hline Tachycardia & $0(0)$ & $1(5.3)$ \\
\hline Agitation & $14^{\mathrm{a}}(20.0)$ & $4(11.2)$ \\
\hline
\end{tabular}

${ }^{a}$ No statistical significances exist between age groups $(p=0.27)$. Agitation was the most common adverse event seen, particularly in infants. 
Table 4 Dexmedetomidine administration for longer or shorter than 72 hours

\begin{tabular}{|c|c|c|c|}
\hline & On dexmedetomidine $<3 \mathrm{~d}$ & On dexmedetomidine $\geq 3 \mathrm{~d}$ & \\
\hline Number $=106$ & 76 & 30 & \\
\hline Characteristics-continuous variables & \multicolumn{2}{|l|}{ Median (range) } & $p$-value \\
\hline Age (mo) & $7.8(0.1-209.4)$ & $3.5(0.1-193.5)$ & 0.002 \\
\hline Extubation after CVICU admission (h) & $20.0(0.5-619.8)$ & $102.8(3.7-457.0)$ & $<0.001$ \\
\hline Characteristics-categorical variables & \multicolumn{2}{|l|}{ Number (\%) } & $p$-value \\
\hline Intubated when dexmedetomidine started & $40(52.6)$ & $29(96.7)$ & $<0.001$ \\
\hline Trisomy 21 & $15(19.7)$ & $4(13.3)$ & 0.43 \\
\hline Other syndromes & $13(17.1)$ & $3(10.0)$ & 0.54 \\
\hline $\begin{array}{l}\text { Adverse response after discontinuing } \\
\text { dexmedetomidine }\end{array}$ & $14(18.4)$ & $9(30.0)$ & 0.19 \\
\hline Withdrawal syndrome & $1(1.3)$ & $5(16.7)$ & 0.007 \\
\hline
\end{tabular}

multimodal analgesia and sedation protocol in 107 infants and children following cardiac surgery. The data seem to suggest that a continuous infusion of this medication appears to be safe and efficacious in this group of children to provide postoperative analgesia and sedation.

The overall median dose of dexmedetomidine in the study group, ranging from 0.8 to $2.17 \mu \mathrm{g} / \mathrm{kg} / \mathrm{h}$, was higher than previously reported and was administered longer than approved by the FDA. ${ }^{2-4}$ In a pharmacokinetic study of dexmedetomidine in infants following cardiac surgery, the authors suggested that the pediatric population may benefit from higher infusion rates per kilogram than the currently approved rate. ${ }^{9,10}$

Despite its widespread clinical utilization in pediatric intensive care units, there is currently only limited evidence with conflicting results to support the safety and efficacy in this age group. ${ }^{11-13}$
The safety profile of dexmedetomidine administration appears favorable in the study group. Despite the higher dose range, patients' vital signs remained steady throughout infusion with the exception of an initial decrease in heart rate from baseline to day 1 . Furthermore, the overall systolic blood pressure and respiratory rates remained stable. Incidences of adverse reactions following initiation of dexmedetomidine were low. In seven patients (6.5\%), dexmedetomidine was discontinued due to observed adverse reactions. The most common adverse reaction was bradycardia, with one due to hypertension. The adverse reactions reported correlate with a higher median bolus dose; therefore, we recommend caution using a bolus dose of $1 \mu \mathrm{g} / \mathrm{kg}$. In fact, most side effects were correlated with administration of boluses in addition to a continuous infusion. As a result, the CVICU has discontinued the use of boluses of the study drug. Children, especially infants who have undergone cardiac surgery, postoperatively

Table 5 Patient characteristics by age group

\begin{tabular}{|c|c|c|c|}
\hline Age groups & $\leq 1$ year old & $>1$ year old & \\
\hline Number (\%) & $70(65 \%)$ & $37(35 \%)$ & \\
\hline Characteristics & \multicolumn{2}{|l|}{ Median (range) } & $p$-value \\
\hline Risk adjustment for congenital heart surgery score & $2(1-6)$ & $3(1-3)$ & 0.82 \\
\hline Dexmedetomidine starting dose $(\mu \mathrm{g} / \mathrm{kg} / \mathrm{h})$ & $0.89(0.21-2.17)$ & $0.5(0.40-1.50)$ & 0.44 \\
\hline Dexmedetomidine bolus given (number) & 11 & 9 & 0.45 \\
\hline Dexmedetomidine bolus dose $(\mu \mathrm{g} / \mathrm{kg})$ & $0.91(0.27-1.14)$ & $0.75(0.70-2.00)$ & 0.13 \\
\hline \multicolumn{4}{|l|}{ On dexmedetomidine time } \\
\hline Days & $1.8(0.04-23.9)$ & $1.2(0-4.0)$ & \multirow[t]{2}{*}{0.008} \\
\hline Hours & $43.5(1-573)$ & $28(0-232)$ & \\
\hline $\begin{array}{l}\text { Time between postoperative CVICU admission and } \\
\text { dexmedetomidine start (h) }\end{array}$ & $20.8(0.3-181.5)$ & $8.3(0-58)$ & 0.003 \\
\hline Time to extubation after postoperative CVICU admission (h) & $46.5(3.7-619.8)$ & $8.4(0.5-135)$ & $<0.001$ \\
\hline
\end{tabular}

Abbreviation: CVICU, cardiovascular intensive care unit. 
rely on their intrinsic heart rate for compensation for postoperative low cardiac output syndrome, and therefore these children could be more sensitive to dexmedetomidine than patients who receive dexmedetomidine as an elective sedation. ${ }^{14}$ Lam et al ${ }^{15}$ in a more recent study reported safe use of dexmedetomidine in patients with heart failure. The recorded inotrope scores in our study reflect a progressively decreasing inotropic need, as would be expected, in a postoperative recovery ( - Fig. 2 ). Lam et al $^{12}$ in a retrospective study described a similar finding in infants, during the first 24 hours postoperatively, while on dexmedetomidine. Hemodynamic instability, based on the documented episodes of hypotension and bradycardia, appears less pronounced in our patient population (6.5\%) than reported previously. ${ }^{4,12}$ Chrysostomou et $\mathrm{al}^{2}$ reported initial decrease in systolic blood pressure in their pediatric study and Hosokawa et $\mathrm{al}^{16}$ reported clinically significant bradycardia or hypotension in $21.4 \%$ of their 141 pediatric patients.

Thirty-eight patients (35.5\%) were extubated and breathing spontaneously at initiation of dexmedetomidine. In these patients, respiratory rates remained stable throughout the continuous infusion, including in the infant group. Median time to extubation for intubated patients was 5.8 hours postinitiation. We did not have a control group, but our institutional median extubation time was 6 hours at the time, which is a comparable number to the median time of our study group. This indicates that dexmedetomidine did not delay extubation.

Our clinical experience that dexmedetomidine, as part of a multimodal analgesia and sedation protocol, is effective and reduces benzodiazepine requirements postoperatively appears to be validated by this study, within the obvious limits of a retrospective analysis. In previous years, we observed several children displaying delirium, especially on midazolam infusions. Subsequently, a goal of the CVICU clinical team was to reduce the use of midazolam. In a prospective trial, Tobias et $\mathrm{al}^{17}$ compared postoperative administration of dexmedetomidine to a midazolam infusion and found that the dexmedetomidine group had more effective sedation and less morphine requirements. The pain and sedation scores appear quite appropriate in our population, and only two children on dexmedetomidine received midazolam. Nearly all children in our study group were started postoperatively on a fentanyl continuous infusion plus patient- or nurse-administered analgesia boluses. Once the children tolerated clear liquids, intravenous fentanyl was usually changed to enteral morphine or oxycodone.

The SBS sedation scores were implemented as a routine assessment tool during the study period, 2010 to 2011, which explains why they were not available for all patients. The goal for most of our postoperative patients is a sedation level between 0 (comfortable, awake) and -1 (asleep, but arousable). In our study, there was a mean of -0.38 (standard deviation 1 ). The FLACC pain scores (with $0=$ no pain, $10=$ worst possible pain) also showed excellent analgesia with mean pain scores of between 1 and 2 out of 10 during the dexmedetomidine administration. The statistically significant increase in pain scores (from 0.88 to 1.38 out of 10 ) pre- and poststudy drug administration might be explained as the cause, rather than the result, of the study drug initiation. In the adult and pediatric literature, there is suggestion to use dexmedetomidine in monotherapy. However, in our postoperative patient population, only children who did not seem to have adequate analgesia and sedation with an opioid alone (usually fentanyl continuous infusion plus nurse-administered analgesia bolus) had dexmedetomidine added to their medication regimen. ${ }^{14,18}$

Prolonged infusions of dexmedetomidine have led to concern over potential withdrawal, defined herein as an adverse reaction postdiscontinuation of dexmedetomidine infusion, consisting of tachycardia and/or hypertension with agitation. ${ }^{19,20}$ More than $20 \%$ of children in the study group displayed irritability or agitation after weaning and discontinuation of dexmedetomidine, which correlated with length of administration. Gupta et $\mathrm{al}^{13}$ noted rebound hypertension and rebound tachycardia even with a lower dose range of 0.1 to $1.5 \mu \mathrm{g} / \mathrm{kg} / \mathrm{h}$ following median duration of 196 hour continuous infusion (range: 131-275 hours). Burbano et $\mathrm{al}^{11}$ described similar symptoms of withdrawal; however, in contrast, this study did not reveal any clinically significant tachyarrhythmias. Additionally, in our patients the rate of isolated tachycardia was rare, unless it occurred in the context of agitation. All three symptoms (tachycardia, hypertension, and agitation) were observed in only three (3\%) patients (two infants and one in older age group). Infants were twice as likely to show agitation following discontinuation of dexmedetomidine compared with the older age groups.

Our clinical practice for children who have been on dexmedetomidine longer than 3 to 5 days is to either wean the medication by $0.1 \mu \mathrm{g} / \mathrm{kg} / \mathrm{h}$ every 12 to 24 hours until off or to administer enteral clonidine 1 to $3 \mu \mathrm{g} / \mathrm{kg} /$ dose every 6 hours and wean off the dexmedetomidine over 24 to 48 hours. The effectiveness of this protocol was beyond the scope of this study. Of note, some children displayed rebound tachycardia, hypertension, and agitation after discontinuation of the study drug of less than 2 days administration, which might be explained by withdrawal and/or less well-controlled pain. The half-life of clonidine is two to three times longer in children than dexmedetomidine; therefore, it is a more suitable agent to be weaned off in patients who are at risk for $\alpha-2$ agonist withdrawal.

Evidence of use of dexmedetomidine in preterm and fullterm neonates is scarce. The nine neonates in our study were all full-term and tolerated dexmedetomidine well, requiring lower median doses than our older infants ( 0.56 vs. $0.77 \mu \mathrm{g} / \mathrm{kg} / \mathrm{h}$ ). Chrysostomou et $\mathrm{al}^{2}$ noted similar findings between their neonatal and infant groups, suggesting immature liver and kidney function in neonates, wherein dexmedetomidine accumulates over time requiring decreasing dose requirements. Our doses were higher than those studied by Chrysostomou et $\mathrm{al}^{10}$ in preterm and term neonates $(0.2-0.5 \mu \mathrm{g} / \mathrm{kg} / \mathrm{h})$ and on par with O'Mara et $\mathrm{al}^{21}$ data on premature infants (mean $0.6 \mu \mathrm{g} / \mathrm{kg} / \mathrm{h}$ [0.3-1.2]). Despite requiring the lowest dose compared with other age groups, neonates exhibited a large decrease in heart rate between baseline and hour 12 . While not clinically considered bradycardia, this is noteworthy for clinicians especially with 
regard to patients who are at risk for low cardiac output syndrome postoperatively. Despite our low statistical power on our nine patients, we felt it was important to describe this group of young patients separately.

There are limited data regarding the postoperative use of dexmedetomidine in children with trisomy 21. In a case study of five infants between 2 and 7 months of age, the study medication appeared to be effective, well tolerated, and useful in weaning off ventilators. ${ }^{22}$ Another study reviewed pediatric opioid use following cardiac surgery in children with trisomy 21 ( $n=44)$ and those without $(n=77)$. There were no differences in opioid administration; however, children with trisomy 21 received significantly less dexmedetomidine postoperatively. ${ }^{23}$ In our study, the 19 children with trisomy 21 did not differ in regard to dosing or length of administration and did not show differences in adverse events or incidence of withdrawal. Overall, the findings in this study suggest that dexmedetomidine may not affect patients with trisomy differently and is overall safe to use in this patient population.

This is a retrospective study without a control group. The samples for the neonate population are not sufficient to reach statistical power. Unlike for opioids and benzodiazepines, validated scales do not exist to quantify withdrawal from dexmedetomidine and some patient files were missing complete SBS sedation scores.

In conclusion, dexmedetomidine appears to be a safe and efficacious agent providing analgesia and sedation as part of a multimodal treatment protocol throughout all pediatric age groups following cardiac surgery. Our findings indicate that higher doses than previously reported may be safe up to $2 \mu \mathrm{g} /$ $\mathrm{kg} / \mathrm{h}$. Validated assessment scores indicate adequate sedation and good analgesia. Following discontinuation, especially after prolonged infusion ( $>72$ hours), patients experienced transient agitation, with few patients experiencing clinically significant withdrawal. Neonates tolerated dexmedetomidine well and safely tolerated extubation on dexmedetomidine. There was no significant difference in overall use and adverse reactions between patients with trisomy 21 and those without. Overall, this study indicates that dexmedetomidine might be a safe option for sedation and analgesia in these patient groups. Further research, especially randomized controlled trials, in the pediatric population is required.

\section{Acknowledgments}

We would like to acknowledge and thank Erin Zielinski and Chai-Wei Lin for their assistance with data management and statistical analysis.

\section{References}

1 Precedex (Dexmedetomidine) [package insert]. Lake Forest, IL: Hospira, Inc.; 2008

2 Chrysostomou C, Sanchez De Toledo J, Avolio T, et al. Dexmedetomidine use in a pediatric cardiac intensive care unit: can we use it in infants after cardiac surgery? Pediatr Crit Care Med 2009; 10(6):654-660
3 Petroz GC, Sikich N, James M, et al. A phase I, two-center study of the pharmacokinetics and pharmacodynamics of dexmedetomidine in children. Anesthesiology 2006;105(6):1098-1110

4 Chrysostomou C, Di Filippo S, Manrique AM, et al. Use of dexmedetomidine in children after cardiac and thoracic surgery. Pediatr Crit Care Med 2006;7(2):126-131

5 Jenkins KJ. Risk adjustment for congenital heart surgery: the RACHS-1 method. Semin Thorac Cardiovasc Surg Pediatr Card Surg Annu 2004;7:180-184

6 Gaies MG, Gurney JG, Yen AH, et al. Vasoactive-inotropic score as a predictor of morbidity and mortality in infants after cardiopulmonary bypass. Pediatr Crit Care Med 2010;11(2): 234-238

7 Merkel SI, Voepel-Lewis T, Shayevitz JR, Malviya S. The FLACC: a behavioral scale for scoring postoperative pain in young children. Pediatr Nurs 1997;23(3):293-297

8 Curley MA, Harris SK, Fraser KA, Johnson RA, Arnold JH. State Behavioral Scale: a sedation assessment instrument for infants and young children supported on mechanical ventilation. Pediatr Crit Care Med 2006;7(2):107-114

9 Su F, Nicolson SC, Gastonguay MR, et al. Population pharmacokinetics of dexmedetomidine in infants after open heart surgery. Anesth Analg 2010;110(5):1383-1392

10 Chrysostomou C, Schulman SR, Herrera Castellanos M, et al. A phase II/III, multicenter, safety, efficacy, and pharmacokinetic study of dexmedetomidine in preterm and term neonates. J Pediatr 2014;164(2):276-82.e1, 3

11 Burbano NH, Otero AV, Berry DE, Orr RA, Munoz RA. Discontinuation of prolonged infusions of dexmedetomidine in critically ill children with heart disease. Intensive Care Med 2012;38(2):300-307

12 Lam F, Bhutta AT, Tobias JD, Gossett JM, Morales L, Gupta P. Hemodynamic effects of dexmedetomidine in critically ill neonates and infants with heart disease. Pediatr Cardiol 2012;33(7):1069-1077

13 Gupta P, Whiteside W, Sabati A, et al. Safety and efficacy of prolonged dexmedetomidine use in critically ill children with heart disease. Pediatr Crit Care Med 2012;13(6):660-666

14 Mason KP, Zurakowski D, Zgleszewski SE, et al. High dose dexmedetomidine as the sole sedative for pediatric MRI. Paediatr Anaesth 2008;18(5):403-411

15 Lam F, Ransom C, Gossett JM, et al. Safety and efficacy of dexmedetomidine in children with heart failure. Pediatr Cardiol 2013; 34(4):835-841

16 Hosokawa K, Shime N, Kato Y, et al. Dexmedetomidine sedation in children after cardiac surgery. Pediatr Crit Care Med 2010;11(1): 39-43

17 Tobias JD, Berkenbosch JW. Sedation during mechanical ventilation in infants and children: dexmedetomidine versus midazolam. South Med J 2004;97(5):451-455

18 Pichot C, Ghignone M, Quintin L. Dexmedetomidine and clonidine: from second- to first-line sedative agents in the critical care setting? J Intensive Care Med 2012;27(4):219-237

19 Iirola T, Aantaa R, Laitio R, et al. Pharmacokinetics of prolonged infusion of high-dose dexmedetomidine in critically ill patients. Crit Care 2011;15(5):R257

20 Bejian S, Valasek C, Nigro JJ, Cleveland DC, Willis BC. Prolonged use of dexmedetomidine in the paediatric cardiothoracic intensive care unit. Cardiol Young 2009;19(1):98-104

21 O'Mara K, Gal P, Wimmer J, et al. Dexmedetomidine versus standard therapy with fentanyl for sedation in mechanically ventilated premature neonates. J Pediatr Pharmacol Ther 2012; 17(3):252-262

22 Kalyanaraman M, Costello JL, Starr JP. Use of dexmedetomidine in patients with trisomy 21 after cardiac surgery. Pediatr Cardiol 2007;28(5):396-399

23 Van Driest SL, Shah A, Marshall MD, et al. Opioid use after cardiac surgery in children with Down syndrome. Pediatr Crit Care Med 2013;14(9):862-868 